\title{
Comparison of three classes of Marginal Risk Set Model in predicting infant mortality among newborn babies at Kigali University Teaching Hospital, Rwanda, 2016
}

\author{
Paul Gatabazi ${ }^{*}$, Sileshi Fanta Melesse and Shaun Ramroop
}

\begin{abstract}
Background: The Infant Mortality Rate (IMR) in Sub-Saharan Africa (SSA) remains the highest relatively to the rest of the world. In the past decade, the policy on reducing infant mortality in SSA was reinforced and both infant mortality and parental death decreased critically for some countries of SSA. The analysis of risk to death or attracting chronic disease may be done for helping medical practitioners and decision makers and for better preventing the infant mortality.

Methods: This study uses popular statistical methods of re-sampling and one selected model of multiple events analysis for measuring the survival outcomes for the infants born in 2016 at Kigali University Teaching Hospital (KUTH) in Rwanda, a country of SSA, amidst maternal and child's socio-economic and clinical covariates. Dataset comprises the newborns with correct information on the covariates of interest. The Bootstrap Marginal Risk Set Model (BMRSM) and Jackknife Marginal Risk Set Model (JMRSM) for the available maternal and child's socioeconomic and clinical covariates were conducted and then compared to the outcome with Marginal Risk Set Model (MRSM). That was for measuring stability of the MRSM.

Results: The 2117 newborns had the correct information on all the covariates, 82 babies died along the study time, 69 stillborn babies were observed while 1966 were censored. Both BMRSM JMRSM and MRSM displayed the close results for significant covariates. The BMRSM displayed in some instance, relatively higher standard errors for nonsignificant covariates and this emphasized their insignificance in MRSM. The models revealed that female babies survive better than male babies. The risk is higher for babies whose parents are under 20 years old parents as compared to other parents' age groups, the risk decreases as the APGAR increases, is lower for underweight babies than babies with normal weight and overweight and is lower for babies with normal circumference of head as compared to those with relatively small head.

Conclusion: The results of JMRSM were closer to MRSM than that of BMRSM. Newborns of mothers aged less than 20 years were at relatively higher risk of dying than those who their mothers were aged 20 years and above. Being abnormal in weight and head increased the risk of infant mortality. Avoidance of teenage pregnancy and provision of clinical care including an adequate dietary intake during pregnancy would reduce the IMR in Kigali.
\end{abstract}

Keywords: Infant mortality, Survival analysis, Marginal risk set model, Re-sampling, Covariate, Rwanda

\footnotetext{
* Correspondence: Gatabazi001@gmail.com

Department of Statistics, University of Kwazulu Natal, Pietermaritzburg,

Private Bag X 01, Scottsville 3209, South Africa
}

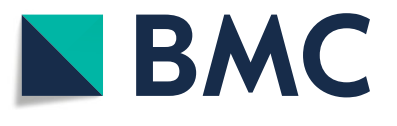

(c) The Author(s). 2020 Open Access This article is distributed under the terms of the Creative Commons Attribution 4.0 International License (http://creativecommons.org/licenses/by/4.0/), which permits unrestricted use, distribution, and

reproduction in any medium, provided you give appropriate credit to the original author(s) and the source, provide a link to the Creative Commons license, and indicate if changes were made. The Creative Commons Public Domain Dedication waiver (http://creativecommons.org/publicdomain/zero/1.0/) applies to the data made available in this article, unless otherwise stated. 


\section{Background}

The discrepancy in IMR and low life expectancy of the SSA versus the other parts of the world attracts several researchers. The report of the World Bank in 2011 pointed that the IMR was 75/1000 in SSA versus 11/1000 in developed countries [1]. The same report pointed that half of the ten million children who die every year is in SSA. The World Bank dataset from 1960 to 2005 suggests that low life expectancy at birth in SSA is relatively higher in Middle Africa as compared to other sub-regional disparities of SSA [2]. The World Bank records of 2017 indicated that the IMR was 51.50/1000 in SSA [3]. Central African Republic had the highest IMR of 87.60/ 1000 , the lowest IMR were found in Mauritius (11.60/1000), the IMR in Rwanda was 28.90/1000. Several studies on factors that could lower the infant mortality have been done and recommendations were suggested but the IMR remains a problem in SSA.

The multiple events model for infant mortality at the Kigali University Teaching Hospital analysed in [4] leaves a question on whether the adopted model is stable. The main causes of instability may be the correlation of the covariates or relatively small sample size [5]. One of the ways of assessing instability in survival regression models is a use of re-sampling techniques [6]. The analysis in [4] is a none re-sampled model that used the primary dataset of the year 2016. Two observable events per subject are death and the occurrence of at least one of the common conditions that may also cause the long-term death to infants. It was found that the Marginal Risk Set Model (MRSM) also known as the Wei, Lin and Weissfeld Model (WLWM) fit the data well. The WLWM is among the multiplicative methods for analysing ordered events found in [7]. Other multiplicative models include the Andersen-Gill Model (AGM) and the Prentice, Williams and Peterson Model (PWPM) [8].

The present study uses two popular nonparametric methods of re-sampling namely bootstrap which is based on the random samples with replacement [9], and jackknife method that is based on sampling by leaving out one observation at time [9]. The size of the sample in [4] is 2117 and the record is effective in the year 2016. The long-term results could be assumed according to the stability potentially observed after re-sampling. Several manuscripts on re-sampling in survival analysis are limited on the re-sampled Cox proportional hazards model and on estimating standard errors of the survival and hazard functions such as in $[6,10-13]$ where bootstrap is involved [13-16]; in which the jackknife is implicated or [17-22] where hazard and survival functions with their respective standard errors are of interest. The present study analyses the bootstrap-based MRSM with
1000 replicates and the jackknife-based MRSM. The results are then compared to that of the MRSM.

\section{Methods \\ Dataset}

The time to event data of 2117 newborns at the KUTH is recorded from the 1st January to the 31st December 2016. At KUTH, all newborns are recorded in registries with all details of parents and clinical outcomes of each newborn. The information in registry provides references on card indexes that provide information on clinical behavior of babies after leaving the hospital. KUTH as a site of interest in this study is a central Hospital where most of complicated childbirths countrywide are transferred. In 2016, KUTH recorded relatively high incidence of stillborn cases (69 stillborn babies or 3.259\%) and relatively high infant mortality rate (3.873\%). Table 1 summarises the information on newborns at KUTH along the study time.

The study is interested on subjects with a correct information on the covariates of interests. The two events per subject are observed namely the death and the incidence of at least one chronic disease or complication such as severe oliguria, severe prematurity, very low birth weight, macrosomia, severe respiratory distress, gastroparesis, hemolytic, trisomy, asphyxia and laparoschisis. Apart from the event status and the time to event, 11 covariates are recorded and subdivided in demographic covariates which include the age and the place of residence for parents; clinical covariates for female parents that include obstetric antecedents, type of childbirth and previous abortion. Clinical covariates for babies include APGAR; gender, number of births at a time, weight, circumference of the head, and height. Table 2 gives a description of the variables of interest.

\section{Statistical methods Marginal risk set model}

Assume that $h\left(t \mid \mathbf{x}_{i}\right)$ is the hazard function of the survival time $T$ given the $p$ fixed covariates $\mathbf{x}_{i}=\left(x_{i 1}, x_{i 2}, \ldots, x_{i p}\right)$. Let $h_{0}(t)$ be the hazard function when $\mathbf{x}_{i}=(0,0, \ldots, 0)$ for all $i$, then

$$
h\left(t \mid \mathbf{x}_{i}\right)=h_{0}(t) \exp \left(\boldsymbol{\beta} \mathbf{x}_{i}\right)
$$

Table 1 Summary on newborns under study

\begin{tabular}{ll}
\hline Total observations & 2117 \\
\hline Deaths during the study time & $82(3.873 \%)$ \\
Stillborn babies & $69(3.259 \%)$ \\
Total events & $151(7.132 \%)$ \\
Censored babies & $1966(92.867 \%)$ \\
\hline
\end{tabular}


Table 2 Description of variables in the dataset on newborns at Kigali University Teaching Hospital (KUTH) during the period 01 January-2016 to 31-December-2016

\begin{tabular}{|c|c|c|}
\hline Variable & Description & Codes/Values/Unit \\
\hline Age & Age of parent & $\begin{array}{l}0=\text { under } 20,1=20 \text { years old to } 34 \text { years old }, 2=35 \text { years } \\
\text { old and above }\end{array}$ \\
\hline Residence & Indicator of the residential area of a parent & $0=$ rural, $1=$ urban \\
\hline Antecedents & Indicator on whether a new born is the first or not & $0=$ Not the first new born, $1=$ first newborn, \\
\hline Abortion & Indicator on whether a parent aborted previously & $0=$ not aborted, 1 = aborted once, $2=$ aborted more than once \\
\hline $\begin{array}{l}\text { Childbirth } \\
\text { Gender }\end{array}$ & Type of childbirth Gender of a newborn & $\begin{array}{l}0=\text { born using ventouse, } 1=\text { born naturally, } 2=\text { born after } \\
\text { surgery } 0=\text { female, } 1=\text { male }\end{array}$ \\
\hline Number & Indicator of the number of births at a time & $0=$ singleton, $1=$ multiple \\
\hline APGAR & $\begin{array}{l}\text { Score of appearance, pulse, grimaces, activity and } \\
\text { respiration of a newborn }\end{array}$ & $\begin{array}{l}0=A P G A R \text { less than } 4 / 10,1=A P G A R \text { from } 4 / 10 \text { to } 6 / 10 \\
2=A P G A R \text { greater or equal to } 7 / 10\end{array}$ \\
\hline Weight & Weight of a newborn & $0=$ under $2500 \mathrm{~g}, 1=2500 \mathrm{~g}$ to $4500 \mathrm{~g}, 2=$ above $4500 \mathrm{~g}$ \\
\hline Head & Head circumference of a newborn & $0=$ below $32 \mathrm{~cm}, 1=32 \mathrm{~cm}$ to $36 \mathrm{~cm}, 2=$ above $36 \mathrm{~cm}$ \\
\hline Height & Height of a new born & $0=$ below $46 \mathrm{~cm}, 1=46 \mathrm{~cm}$ to $54 \mathrm{~cm}, 2=$ above $54 \mathrm{~cm}$ \\
\hline Time & Time from recruitment to study termination & Days \\
\hline Event & $\begin{array}{l}\text { Indicator describing if death occurred during the } \\
\text { study time or not }\end{array}$ & $0=$ censored, $1=$ dead \\
\hline$n$ events & Indicator on the rank of records per subject & $1=$ first record, 2 = second record \\
\hline
\end{tabular}

where $\boldsymbol{\beta}=\left(\beta_{1}, \beta_{2}, \ldots, \beta_{p}\right)^{\prime}$ is a $p$-dimensional vector of model parameters [23]. Define an indicator function as.

$\delta_{i j}(t)=1$ if individual $i$ is at risk of the $j^{\text {th }}$ event and $\delta_{i j}(t)=0$ otherwise.

The marginal risk set model (MRSM) or the Wei Lin and Weisfeld Model (WLWM) assumes that events are unordered where each event has its own stratum and each data point appears in all strata [4, 24]. In other words, the $k^{\text {th }}$ time interval per subject is in the $k^{\text {th }}$ stratum, $k=1,2, \ldots, n$.

The hazard function for the $j^{\text {th }}$ event for the individual $i$ is given by

$$
h\left(t \mid \mathbf{x}_{i}\right)=\delta_{i j}(t) h_{0 j}(t) \exp \left(\boldsymbol{\beta}_{j} \mathbf{x}_{i}\right)
$$

\section{Maximum likelihood and parameter estimation}

Let]0, $\tau_{i}$ [be the interval of time in which the individual $i$ is observed with $n_{i}$ the number of events of the individual $i$ along]0, $\tau_{i}$ [and Assume that two events cannot occur simultaneously in continuous time. The probability density function for the outcome $n_{i}$ along] $0, \tau_{i}$ [is given by.

$$
L(\boldsymbol{\Phi})=\prod_{i=1}^{n} L_{i}(\phi)
$$

where

$$
L_{i}(\phi)=\prod_{j=1}^{n_{i}} h\left(t \mid x_{i}\right) e^{-\int_{0}^{\tau_{i}} \delta_{i j}(v) h\left(v \mid x_{i}\right) d v}
$$

In (3), individual $i$ has $n_{i}$ events with $n_{i} \geq 0$ at times $t_{i 1} \leq \mathrm{t}_{i 2} \leq \cdots \leq \mathrm{t}_{\text {ini }}$.

The appropriate partial likelihood functions for tied time to event data is well described in [24] and in [25] and include Breslow's, Efron's and Cox's techniques. The maximum likelihood estimates are given by a system

$$
\left\{\frac{\frac{\partial \ln L(\Phi)}{\partial \alpha}}{\frac{\partial \ln L(\Phi)}{\partial \beta}}\right.
$$

where $\alpha$ is known as the baseline parameter vector while $\beta$ is a vector of model parameters. The NewtonRaphson method is one of numerical methods used for solving system (4). The adequacy checking of the likelihood estimates is done by finding the elements $\mathfrak{I}_{\alpha \alpha}, \mathfrak{I}_{\alpha \beta}$, $\mathfrak{I}_{\beta \alpha}$ and $\mathfrak{J}_{\beta \beta}$ of the information matrix $\mathfrak{I}$ and assume that as $n \rightarrow \infty, \hat{\Phi}-\Phi \mapsto N\left(0, \mathfrak{J}^{-1}(\hat{\Phi})\right)[4,26]$.

In MRSM, $n$ is assumed to be the maximum number of events per subject while $\tau_{k}, k=1,2, \ldots n$ are times to events per subject along the study time with range $[0, T]$. The study time is partitioned into $n+1$ intervals of the form

$$
0-\tau_{1}, 0-\tau_{2}, \ldots, 0-\tau_{n}, 0-T .
$$

STATA 15 provides results of the MRSM by applying the Cox Proportional Hazards Model (CPHM) to the 
dataset in the setup (5). The test of proportional hazards assumption is done by checking patterns of survival functions per groups of each covariate. Figure 1 presents the patterns of survival functions per groups of each covariate using Kaplan-Meier estimation. The patterns are approximately parallel for the covariates of interest. This allows a construction of the MRSM for all the covariates.

\section{Re-sampled MRSM}

The Bootstrap Marginal Risk set Model (BMRSM) is the inference of model (2) based on bootstrap samples (see Appendix). The BMRSM consists of applying model (2) to each of the $B$ bootstrap samples $\mathbf{x}_{i}{ }^{* k}, \forall k \in[1, B]$ of covariates $\mathbf{x}_{i}, \forall i \in[1, p]$. Bootstrap model parameter estimation in presence of tied events uses either Breslow, Efron or Cox approach. The bootstrap standard error is obtained by using Eq. (6) of the Appendix.

As for the BMRSM, the Jackknife Marginal Risk Model (JMRSM) consists of applying model (2) to each of the $n$ jackknife samples $\mathbf{x}_{i}{ }^{* k}$ of covariates $\mathbf{x}_{i}, i$ $\in[1, p]$ with a use of Breslow, Efron or Cox approach for estimating the jackknife model parameters. The Jackknife standard error is given by Eq. (7) found in the Appendix.

\section{Results}

Using Breslow estimation [27], Table 3 presents unadjusted MRSM, BMRSM, JMRSM and corresponding adjusted models. Unadjusted and adjusted MRSM, BMRSM and JMRSM are also presented in Tables 4 and 5 for Efron [28] and Cox estimation [29].

The results of the unadjusted JMRSM are relatively close to that of the unadjusted MRSM (Table 3). The standard errors in JMRSM and MRSM are close for all covariates. The standard errors in BMRSM and MRSM are also close for covariates except for all levels of covariates childbirth where the standard error in BMRSM is about 4 times that of MRSM and the upper levels of covariates weight, head and height where the standard error in BMRSM is about 20 times that of MRSM. Significance difference in levels of covariates is found at the same covariates for both MRSM, BMRSM and JMRSM except at the upper

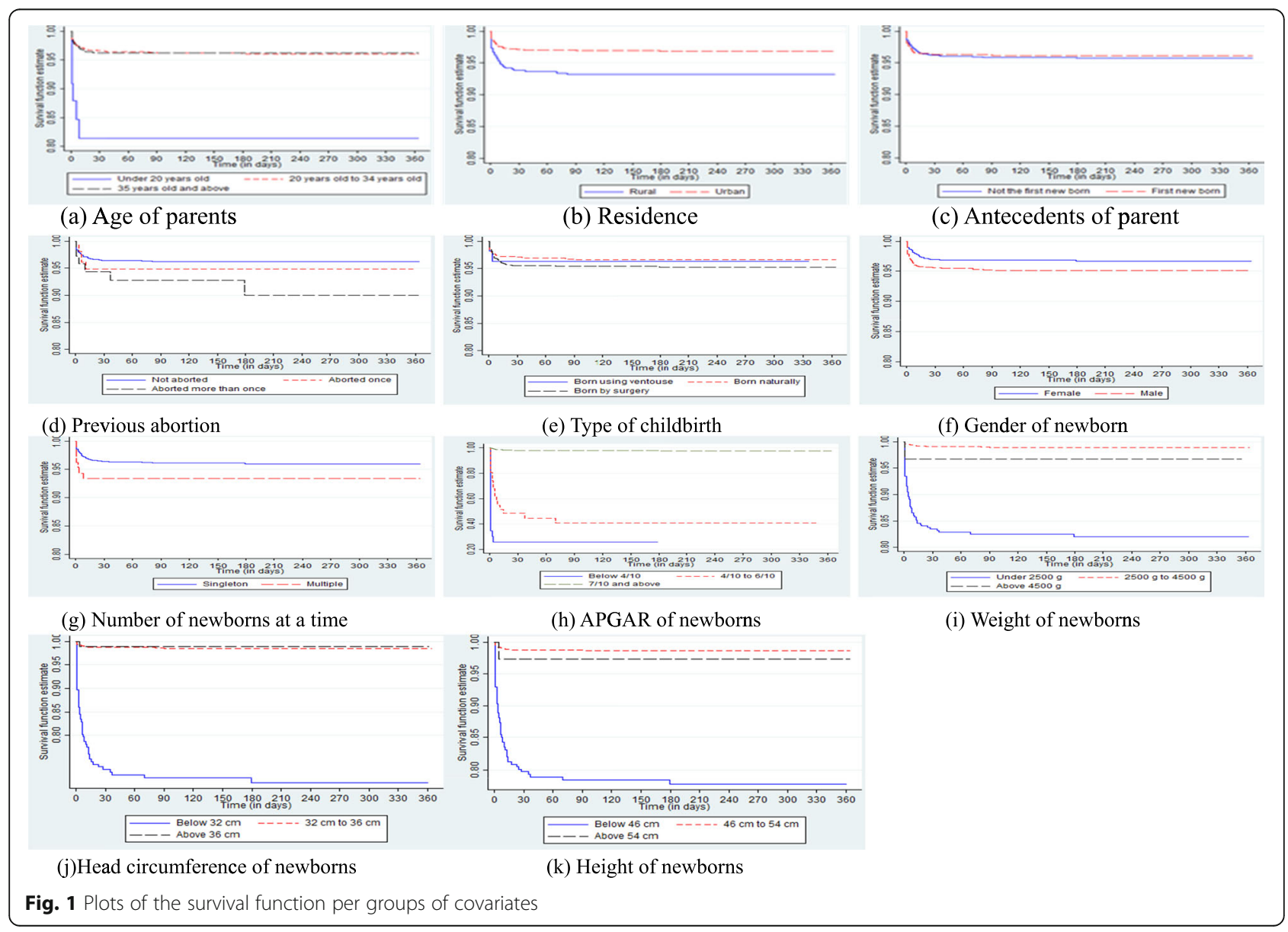


Table 3 Breslow estimation

\begin{tabular}{|c|c|c|c|c|c|c|c|c|c|c|c|c|c|}
\hline \multicolumn{6}{|l|}{ MRSM } & \multicolumn{4}{|c|}{ BMRSM } & \multicolumn{4}{|c|}{ JMRSM } \\
\hline $\begin{array}{l}\text { Covariate } \\
\text { (reference) }\end{array}$ & Level & $\mathrm{HR}$ & SE & $P>z$ & $\begin{array}{l}95 \% \\
\mathrm{Cl}\end{array}$ & $\mathrm{HR}$ & SE & $P>z$ & $95 \% \mathrm{Cl}$ & $\mathrm{HR}$ & SE & $P>z$ & $95 \% \mathrm{Cl}$ \\
\hline \multirow[t]{2}{*}{$\begin{array}{l}\text { Age (Under } 20 \\
\text { years old) }\end{array}$} & $\begin{array}{l}20 \text { to } 34 \\
\text { years old }\end{array}$ & 0.277 & 0.100 & $p<0.001$ & $\begin{array}{l}{[0.137} \\
0.560]\end{array}$ & 0.277 & 0.088 & $p<0.001$ & {$[0.149 ; 0.515]$} & 0.277 & 0.081 & $p<0.001$ & $\begin{array}{l}{[0.155 ;} \\
0.493]\end{array}$ \\
\hline & $\begin{array}{l}35 \text { years old } \\
\text { and above }\end{array}$ & 0.395 & 0.157 & 0.020 & $\begin{array}{l}{[0.181} \\
0.863]\end{array}$ & 0.395 & 0.132 & 0.005 & {$[0.205 ; 0.761]$} & 0.395 & 0.127 & 0.004 & $\begin{array}{l}{[0.210 ;} \\
0.741]\end{array}$ \\
\hline Residence (Rural) & Urban & 0.847 & 0.139 & 0.309 & $\begin{array}{l}{[0.614 ;} \\
1.167]\end{array}$ & 0.847 & 0.148 & 0.341 & {$[0.601 ; 1.193]$} & 0.847 & 0.158 & 0.372 & $\begin{array}{l}{[0.587 ;} \\
1.220]\end{array}$ \\
\hline $\begin{array}{l}\text { Antecedents (Not } \\
1 \text { st newborn) }\end{array}$ & 1st newborn & 0.806 & 0.157 & 0.270 & $\begin{array}{l}{[0.550 ;} \\
1.182]\end{array}$ & 0.806 & 0.138 & 0.207 & {$[0.577 ; 1.126]$} & 0.806 & 0.134 & 0.193 & $\begin{array}{l}{[0.582 ;} \\
1.116]\end{array}$ \\
\hline \multirow[t]{2}{*}{$\begin{array}{l}\text { Abortion (Not } \\
\text { aborted) }\end{array}$} & Aborted once & 1.405 & 0.398 & 0.231 & $\begin{array}{l}{[0.806 ;} \\
2.448]\end{array}$ & 1.405 & 0.459 & 0.298 & {$[0.741 ; 2.664]$} & 1.405 & 0.471 & 0.311 & $\begin{array}{l}{[0.728 ;} \\
2.710]\end{array}$ \\
\hline & $\begin{array}{l}\text { Aborted more } \\
\text { than once }\end{array}$ & 0.479 & 0.161 & 0.028 & $\begin{array}{l}{[0.248 ;} \\
0.925]\end{array}$ & 0.479 & 0.280 & 0.208 & {$[0.152 ; 1.507]$} & 0.479 & 0.360 & 0.328 & $\begin{array}{l}{[0.110 ;} \\
2.094]\end{array}$ \\
\hline \multirow[t]{2}{*}{$\begin{array}{l}\text { Childbirth } \\
\text { (Ventouse) }\end{array}$} & Natural & 0.873 & 0.491 & 0.808 & $\begin{array}{l}{[0.290 ;} \\
2.627]\end{array}$ & 0.873 & 1.973 & 0.952 & {$[0.010 ; 73.427]$} & 0.873 & 0.329 & 0.718 & $\begin{array}{l}{[0.416 ;} \\
1.829]\end{array}$ \\
\hline & Surgery & 1.115 & 0.613 & 0.843 & $\begin{array}{l}{[0.380 ;} \\
3.274]\end{array}$ & 1.115 & 2.517 & 0.962 & {$[0.013 ; 93.040]$} & 1.115 & 0.372 & 0.744 & $\begin{array}{l}{[0.580 ;} \\
2.143]\end{array}$ \\
\hline Gender (Female) & Male & 1.740 & 0.296 & 0.001 & $\begin{array}{l}{[1.247 ;} \\
2.429]\end{array}$ & 1.740 & 0.324 & 0.003 & {$[1.209 ; 2.505]$} & 1.740 & 0.337 & 0.004 & $\begin{array}{l}{[1.191 ;} \\
2.544]\end{array}$ \\
\hline $\begin{array}{l}\text { Number } \\
\text { (Singleton) }\end{array}$ & Multiple & 0.409 & 0.131 & 0.005 & $\begin{array}{l}{[0.218 ;} \\
0.766]\end{array}$ & 0.409 & 0.107 & 0.001 & {$[0.245 ; 0.682]$} & 0.409 & 0.100 & $p<0.001$ & $\begin{array}{l}{[0.252 ;} \\
0.661]\end{array}$ \\
\hline \multirow[t]{2}{*}{$\begin{array}{l}\text { APGAR (Below 4/ } \\
\text { 10) }\end{array}$} & $4 / 10$ to $6 / 10$ & 0.377 & 0.112 & 0.001 & $\begin{array}{l}{[0.211 ;} \\
0.673]\end{array}$ & 0.377 & 0.127 & 0.004 & {$[0.195 ; 0.729]$} & 0.377 & 0.139 & 0.008 & $\begin{array}{l}{[0.182 ;} \\
0.778]\end{array}$ \\
\hline & $\begin{array}{l}\text { 7/10 and } \\
\text { above }\end{array}$ & 0.130 & 0.036 & $p<0.001$ & $\begin{array}{l}{[0.076 ;} \\
0.222]\end{array}$ & 0.130 & 0.033 & $p<0.001$ & {$[0.079 ; 0.212]$} & 0.130 & 0.031 & $p<0.001$ & $\begin{array}{l}{[0.081 ;} \\
0.208]\end{array}$ \\
\hline \multirow[t]{2}{*}{$\begin{array}{l}\text { Weight (Under } \\
2500 \mathrm{~g} \text { ) }\end{array}$} & $\begin{array}{l}2500 \mathrm{~g} \text { to } 4500 \\
\mathrm{~g}\end{array}$ & 0.250 & 0.068 & $p<0.001$ & $\begin{array}{l}{[0.146 ;} \\
0.427]\end{array}$ & 0.250 & 0.064 & $p<0.001$ & {$[0.151 ; 0.412]$} & 0.250 & 0.063 & $p<0.001$ & $\begin{array}{l}{[0.153 ;} \\
0.408]\end{array}$ \\
\hline & Above $4500 \mathrm{~g}$ & 0.442 & 0.285 & 0.206 & $\begin{array}{l}{[0.125} \\
1.565]\end{array}$ & 0.442 & 4.002 & 0.928 & $\begin{array}{l}{[0.000 ; 2.290 \times} \\
\left.10^{7}\right]\end{array}$ & 0.442 & 0.508 & 0.478 & $\begin{array}{l}{[0.046 ;} \\
4.222\end{array}$ \\
\hline \multirow[t]{2}{*}{$\begin{array}{l}\text { Head (Below } 32 \\
\mathrm{~cm} \text { ) }\end{array}$} & $32 \mathrm{~cm}$ to $36 \mathrm{~cm}$ & 0.456 & 0.128 & 0.005 & $\begin{array}{l}{[0.263 ;} \\
0.789]\end{array}$ & 0.456 & $\begin{array}{l}0.115 \\
0\end{array}$ & 0.002 & {$[0.277 ; 0.749]$} & 0.456 & 0.117 & 0.002 & $\begin{array}{l}{[0.275 ;} \\
0.753]\end{array}$ \\
\hline & Above $36 \mathrm{~cm}$ & 0.290 & 0.219 & 0.102 & $\begin{array}{l}{[0.066 ;} \\
1.278]\end{array}$ & 0.290 & 4.156 & 0.931 & $\begin{array}{l}{[0.000 ; 4.470 \times} \\
\left.10^{11}\right]\end{array}$ & 0.290 & 0.284 & 0.206 & $\begin{array}{l}{[0.043 ;} \\
1.971]\end{array}$ \\
\hline \multirow[t]{2}{*}{$\begin{array}{l}\text { Height (Below } \\
36 \mathrm{~cm} \text { ) }\end{array}$} & $46 \mathrm{~cm}$ to $54 \mathrm{~cm}$ & 0.894 & 0.276 & 0.716 & $\begin{array}{l}{[0.488 ;} \\
1.637]\end{array}$ & 0.894 & 0.241 & 0.677 & {$[0.527 ; 1.516]$} & 0.894 & 0.253 & 0.692 & $\begin{array}{l}{[0.513 ;} \\
1.557]\end{array}$ \\
\hline & Above $54 \mathrm{~cm}$ & 1.670 & 1.264 & 0.498 & $\begin{array}{l}{[0.379 ;} \\
7.361]\end{array}$ & 1.670 & 22.884 & 0.970 & $\begin{array}{l}{[0.000 ; 7.73 \times} \\
\left.10^{11}\right]\end{array}$ & 1.670 & 1.612 & 0.596 & $\begin{array}{l}{[0.251 ;} \\
11.093]\end{array}$ \\
\hline \multicolumn{6}{|c|}{ Adjusted MRSM } & \multicolumn{4}{|c|}{ Adjusted BMRSM } & \multicolumn{4}{|c|}{ Adjusted JMRSM } \\
\hline $\begin{array}{l}\text { Covariate } \\
\text { (reference) }\end{array}$ & Level & $\mathrm{HR}$ & SE & $P>z$ & $\begin{array}{l}95 \% \\
\mathrm{Cl}\end{array}$ & $\mathrm{HR}$ & SE & $P>z$ & $95 \% \mathrm{Cl}$ & $\mathrm{HR}$ & SE & $P>z$ & $95 \% \mathrm{Cl}$ \\
\hline \multirow[t]{2}{*}{$\begin{array}{l}\text { Age (Under } \\
20 \text { years old) }\end{array}$} & $\begin{array}{l}20 \text { to } 34 \\
\text { years old }\end{array}$ & 0.307 & 0.107 & 0.001 & $\begin{array}{l}{[0.155 ;} \\
0.609]\end{array}$ & 0.309 & 0.089 & $p<0.001$ & {$[0.176 ; 0.543]$} & 0.309 & 0.083 & $p<0.001$ & $\begin{array}{l}{[0.182 ;} \\
0.523]\end{array}$ \\
\hline & $\begin{array}{l}35 \text { years old } \\
\text { and above }\end{array}$ & 0.472 & 0.179 & 0.047 & $\begin{array}{l}{[0.225} \\
0.992]\end{array}$ & 0.489 & 0.145 & 0.016 & {$[0.274 ; 0.874]$} & 0.489 & 0.137 & 0.011 & $\begin{array}{l}{[0.282 ;} \\
0.848]\end{array}$ \\
\hline \multirow[t]{2}{*}{$\begin{array}{l}\text { Abortion (Not } \\
\text { aborted) }\end{array}$} & Aborted once & 1.482 & 0.406 & 0.152 & $\begin{array}{l}{[0.866 ;} \\
2.537]\end{array}$ & - & - & - & - & - & - & - & - \\
\hline & $\begin{array}{l}\text { Aborted more } \\
\text { than once }\end{array}$ & 0.541 & 0.175 & 0.057 & $\begin{array}{l}{[0.287} \\
1.019]\end{array}$ & 1.607 & - 0.304 & -0.012 & - [1.109; 2.328] & - & - & - & - \\
\hline Gender (Female) & Male & 1.672 & 0.280 & 0.002 & $\begin{array}{l}{[1.204 ;} \\
2.321]\end{array}$ & 0.417 & 0.106 & 0.001 & {$[0.254 ; 0.686]$} & 1.607 & 0.316 & 0.016 & $\begin{array}{l}{[1.093 ;} \\
2.363]\end{array}$ \\
\hline $\begin{array}{l}\text { Number } \\
\text { (Singleton) }\end{array}$ & Multiple & 0.401 & 0.128 & 0.004 & $\begin{array}{l}{[0.214 ;} \\
0.750]\end{array}$ & 0.412 & 0.137 & 0.008 & {$[0.215 ; 0.791]$} & 0.417 & 0.103 & $p<0.001$ & $\begin{array}{l}{[0.258 ;} \\
0.677]\end{array}$ \\
\hline
\end{tabular}


Table 3 Breslow estimation (Continued)

\begin{tabular}{|c|c|c|c|c|c|c|c|c|c|c|c|c|c|}
\hline \multirow{2}{*}{$\begin{array}{l}\text { MRSM } \\
\text { APGAR (Below } \\
4 / 10)\end{array}$} & \multirow[b]{2}{*}{$4 / 10$ to $6 / 10$} & \multirow[b]{2}{*}{0.414} & \multirow[b]{2}{*}{0.119} & \multirow[b]{2}{*}{0.002} & \multirow[b]{2}{*}{$\begin{array}{l}{[0.236 ;} \\
0.726]\end{array}$} & \multicolumn{4}{|c|}{ BMRSM } & \multicolumn{4}{|c|}{ JMRSM } \\
\hline & & & & & & 0.150 & 0.034 & $p<0.001$ & {$[0.096 ; 0.234]$} & 0.412 & 0.142 & 0.010 & $\begin{array}{l}{[0.210 ;} \\
0.809]\end{array}$ \\
\hline & $\begin{array}{l}7 / 10 \text { and } \\
\text { above }\end{array}$ & 0.144 & 0.038 & $p<0.001$ & $\begin{array}{l}{[0.086 ;} \\
0.242]\end{array}$ & 0.240 & 0.057 & $p<0.001$ & {$[0.151 ; 0.381]$} & 0.150 & 0.033 & $p<0.001$ & $\begin{array}{l}{[0.098 ;} \\
0.232]\end{array}$ \\
\hline \multirow[t]{2}{*}{$\begin{array}{l}\text { Weight (Under } \\
2500 \mathrm{~g} \text { ) }\end{array}$} & $\begin{array}{l}2500 \mathrm{~g} \text { to } \\
4500 \mathrm{~g}\end{array}$ & 0.238 & 0.060 & $p<0.001$ & $\begin{array}{l}{[0.144 ;} \\
0.391\end{array}$ & 0.478 & 4.519 & 0.938 & {$\left[0.000 ; 5.32 \times 10^{7}\right]$} & 0.240 & 0.057 & $p<0.001$ & $\begin{array}{l}{[0.151 ;} \\
0.381]\end{array}$ \\
\hline & Above $4500 \mathrm{~g}$ & 0.447 & 0.284 & 0.205 & $\begin{array}{l}{[0.129 ;} \\
1.550\end{array}$ & 0.439 & 0.103 & $p<0.001$ & {$[0.277 ; 0.696]$} & 0.478 & 0.419 & 0.400 & $\begin{array}{l}{[0.086 ;} \\
2.669]\end{array}$ \\
\hline \multirow[t]{3}{*}{$\begin{array}{l}\text { Head (Below } \\
32 \mathrm{~cm} \text { ) }\end{array}$} & $32 \mathrm{~cm}$ to $36 \mathrm{~cm}$ & 0.420 & 0.100 & $p<0.001$ & $\begin{array}{l}{[0.264 ;} \\
0.669]\end{array}$ & 0.303 & 4.200 & 0.931 & $\begin{array}{l}{[0.000 ;} \\
\left.1.970 \times 10^{11}\right]\end{array}$ & 0.439 & 0.107 & 0.001 & $\begin{array}{l}{[0.273 ;} \\
0.707]\end{array}$ \\
\hline & Above $36 \mathrm{~cm}$ & 0.284 & 0.210 & 0.089 & $\begin{array}{l}{[0.067 ;} \\
1.211]\end{array}$ & & & & & 0.303 & 0.298 & 0.225 & $\begin{array}{l}{[0.044 ;} \\
2.084]\end{array}$ \\
\hline & & \multicolumn{4}{|c|}{$x^{2}=213.161, p<0.001$} & \multicolumn{4}{|c|}{$x^{2}=203.14, p<0.001$} & \multicolumn{4}{|c|}{$x^{2}=22.310, p<0.001$} \\
\hline
\end{tabular}

level of the covariate abortion where significance is suggested by the MRSM. Following the recommendations of Parzen and Lipsitz [30], the $\chi^{2}$ test statistics suggest a higher performance of the JCPHM as compared to the CPHM and ВСРHM since the $\chi^{2}$ is relatively everywhere lower for the JCPHM..

\section{Discussion}

The overall results of MRSM, BMRSM and JMRSM by different approaches of ties handling (Tables 3, 4 and 5) are not critically different as expected. The STATA default method (Breslow) is then of interest in the analysis. The JMRSM is adopted for checking stability since the results are closer to that of MRSM than that of BMRSM. The similarity between MRSM and JMRSM suggests that the MRSM may be stable. The global analysis upholds the significance difference of all levels of covariates age, gender, number and $A P G A R$ and intermediate levels of covariates weight and head.

The re-sampled adjusted models by Breslow technique of handling tied events suggest that the risk of death or attracting a chronic disease of babies whose parents' age range from 20 to 34 years old is lower than that of babies whose parents are under 20 years old and that of babies whose parents are 35 years and above. Basinga et al. [31] argue that the unintended pregnancy induces abortion in Rwanda, their study suggests a relatively higher rate of teenage unintended pregnancies as compared to the other age ranges, this contributes on the first hand, to the increase of infant mortality rate. On the second hand, the study by Olausson et al. [32] confirms a relatively higher risk for teenage pregnancies due to biological immaturity. As for the advanced maternal age, Lampinen et al. [33] point that it is associated with relatively poorer outcomes to pregnancies due to the observed higher incidence of chronic medical conditions among older women.

The results show that the risk for male babies is higher than that of female babies. This complies with the usual better survival outcome of the females as reports several manuscripts such as [34] or [35]. Multiple babies survive better than singleton babies; this is however against the results from studies conducted in Sub-Saharan Africa by Monden and Smits [36] and Pongou et al. [37]. This may be due to the small number of multiple newborns recorded at KUTH along the year 2016. The survival outcomes of babies whose APGAR is below 4/10 are poorer than that of babies with higher APGAR score. Babies whose weight range from $2500 \mathrm{~g}$ to $4500 \mathrm{~g}$ survive better than those whose weight is below $2500 \mathrm{~g}$ and those whose weight is above $4500 \mathrm{~g}$ while babies whose circumference of head range from $32 \mathrm{~cm}$ to $36 \mathrm{~cm}$ survive better than those whose circumference of head is below $32 \mathrm{~cm}$. The results of APGAR, weight and circumference of the head comply with the recommendations of the clinical medicine and related manuscripts such as [38] for example.

The study shows that the BMRSM is close to JMRSM and MRSM for all significant covariate but the BMRSM shows relatively higher standard errors for some non-significant covariates. The discrepancy between standard errors after re-sampling for covariates such as childbirth, weight, head and height suggests the instability of the MRSM at these specific covariates and this emphasizes their non-significance in the MRSM.

The present analysis is limited on eleven covariates. Unavailable covariates concerning parents that could improve models are, for example, demographic 
Table 4 Efron estimation

\begin{tabular}{|c|c|c|c|c|c|c|c|c|c|c|c|c|c|}
\hline \multicolumn{6}{|l|}{ MRSM } & \multicolumn{4}{|l|}{ BMRSM } & \multicolumn{4}{|c|}{ JMRSM } \\
\hline $\begin{array}{l}\text { Covariate } \\
\text { (reference) }\end{array}$ & Level & $\mathrm{HR}$ & SE & $P>z$ & $95 \% \mathrm{Cl}$ & $\mathrm{HR}$ & SE & $P>z$ & $95 \% \mathrm{Cl}$ & $\mathrm{HR}$ & SE & $P>z$ & $95 \% \mathrm{Cl}$ \\
\hline \multirow[t]{2}{*}{$\begin{array}{l}\text { Age (Under } \\
20 \text { years old) }\end{array}$} & $\begin{array}{l}20 \text { to } 34 \\
\text { years old }\end{array}$ & 0.230 & 0.083 & $p<0.001$ & $\begin{array}{l}{[0.114 ;} \\
0.466]\end{array}$ & 0.230 & 0.086 & $p<0.001$ & $\begin{array}{l}{[0.111 ;} \\
0.478]\end{array}$ & 0.230 & 0.083 & $p<0.001$ & $\begin{array}{l}{[0.114 ;} \\
0.466]\end{array}$ \\
\hline & $\begin{array}{l}35 \text { years old } \\
\text { and above }\end{array}$ & 0.324 & 0.129 & 0.005 & $\begin{array}{l}{[0.149 ;} \\
0.706]\end{array}$ & 0.324 & 0.128 & 0.004 & $\begin{array}{l}{[0.149 ;} \\
0.703]\end{array}$ & 0.324 & 0.125 & 0.004 & $\begin{array}{l}{[0.152 ;} \\
0.691]\end{array}$ \\
\hline Residence (Rural) & Urban & 0.831 & 0.137 & 0.261 & $\begin{array}{l}{[0.602 ;} \\
1.147]\end{array}$ & 0.831 & 0.160 & 0.337 & $\begin{array}{l}{[0.570 ;} \\
1.212]\end{array}$ & 0.831 & 0.174 & 0.376 & $\begin{array}{l}{[0.552 ;} \\
1.252]\end{array}$ \\
\hline $\begin{array}{l}\text { Antecedents (Not } \\
1 \text { st newborn) }\end{array}$ & 1st newborn & 0.756 & 0.149 & 0.156 & $\begin{array}{l}{[0.513 ;} \\
1.113]\end{array}$ & 0.756 & 0.149 & 0.155 & $\begin{array}{l}{[0.514 ;} \\
1.112]\end{array}$ & 0.756 & 0.143 & 0.140 & $\begin{array}{l}{[0.521 ;} \\
1.096]\end{array}$ \\
\hline \multirow[t]{2}{*}{$\begin{array}{l}\text { Abortion (Not } \\
\text { aborted) }\end{array}$} & Aborted once & 1.393 & 0.396 & 0.244 & $\begin{array}{l}{[0.798 ;} \\
2.430]\end{array}$ & 1.393 & 0.470 & 0.326 & $\begin{array}{l}{[0.719 ;} \\
2.699]\end{array}$ & 1.393 & 0.522 & 0.377 & $\begin{array}{l}{[0.668 ;} \\
2.904]\end{array}$ \\
\hline & $\begin{array}{l}\text { Aborted more } \\
\text { than once }\end{array}$ & 0.452 & 0.154 & 0.020 & $\begin{array}{l}{[0.232 ;} \\
0.880]\end{array}$ & 0.452 & 0.322 & 0.265 & $\begin{array}{l}{[0.112 ;} \\
1.826]\end{array}$ & 0.452 & 0.391 & 0.359 & $\begin{array}{l}{[0.083 ;} \\
2.465]\end{array}$ \\
\hline \multirow[t]{2}{*}{$\begin{array}{l}\text { Childbirth } \\
\text { (Ventouse) }\end{array}$} & Natural & 0.736 & 0.408 & 0.580 & $\begin{array}{l}{[0.249 ;} \\
2.179]\end{array}$ & 0.736 & 1.482 & 0.879 & $\begin{array}{l}{[0.014 ;} \\
38.109]\end{array}$ & 0.736 & 0.336 & 0.502 & $\begin{array}{l}{[0.301 ;} \\
1.801]\end{array}$ \\
\hline & Surgery & 0.921 & 0.499 & 0.880 & $\begin{array}{l}{[0.319 ;} \\
2.661]\end{array}$ & 0.921 & 1.858 & 0.968 & $\begin{array}{l}{[0.018 ;} \\
47.963]\end{array}$ & 0.921 & 0.388 & 0.846 & $\begin{array}{l}{[0.403 ;} \\
2.104]\end{array}$ \\
\hline Gender (Female) & Male & 1.823 & 0.312 & $p<0.001$ & $\begin{array}{l}{[1.304 ;} \\
2.549]\end{array}$ & 1.823 & 0.361 & 0.002 & $\begin{array}{l}{[1.238 ;} \\
2.687]\end{array}$ & 1.823 & 0.400 & 0.006 & $\begin{array}{l}{[1.186 ;} \\
2.804]\end{array}$ \\
\hline Number (Singleton) & Multiple & 0.324 & 0.106 & 0.001 & $\begin{array}{l}{[0.170 ;} \\
0.617]\end{array}$ & 0.324 & 0.100 & $p<0.001$ & $\begin{array}{l}{[0.177 ;} \\
0.591]\end{array}$ & 0.324 & 0.096 & $p<0.001$ & $\begin{array}{l}{[0.181 ;} \\
0.578]\end{array}$ \\
\hline \multirow[t]{2}{*}{$\begin{array}{l}\text { APGAR (Below } \\
4 / 10 \text { ) }\end{array}$} & $4 / 10$ to $6 / 10$ & 0.214 & 0.065 & $p<0.001$ & $\begin{array}{l}{[0.118 ;} \\
0.387]\end{array}$ & 0.214 & 0.080 & $p<0.001$ & $\begin{array}{l}{[0.102 ;} \\
0.447]\end{array}$ & 0.214 & 0.093 & $p<0.001$ & $\begin{array}{l}{[0.091 ;} \\
0.501]\end{array}$ \\
\hline & $\begin{array}{l}\text { 7/10 and } \\
\text { above }\end{array}$ & 0.070 & 0.020 & $p<0.001$ & $\begin{array}{l}{[0.041 ;} \\
0.121]\end{array}$ & 0.070 & 0.019 & $p<0.001$ & $\begin{array}{l}{[0.041 ;} \\
0.120\end{array}$ & 0.070 & 0.019 & $p<0.001$ & $\begin{array}{l}{[0.041 ;} \\
0.119]\end{array}$ \\
\hline \multirow[t]{2}{*}{$\begin{array}{l}\text { Weight (Under } \\
2500 \mathrm{~g} \text { ) }\end{array}$} & $\begin{array}{l}2500 \mathrm{~g} \text { to } \\
4500 \mathrm{~g}\end{array}$ & 0.231 & 0.063 & $p<0.001$ & $\begin{array}{l}{[0.135} \\
0.395]\end{array}$ & 0.231 & 0.064 & $p<0.001$ & $\begin{array}{l}{[0.134 ;} \\
0.396]\end{array}$ & 0.231 & 0.062 & $p<0.001$ & $\begin{array}{l}{[0.136 ;} \\
0.391]\end{array}$ \\
\hline & Above $4500 \mathrm{~g}$ & 0.412 & 0.269 & 0.174 & $\begin{array}{l}{[0.115} \\
1.479]\end{array}$ & 0.412 & 3.892 & 0.925 & $\begin{array}{l}{[0.000 ;} \\
\left.4.57 \times 10^{7}\right]\end{array}$ & 0.412 & 0.485 & 0.451 & $\begin{array}{l}{[0.041 ;} \\
4.149]\end{array}$ \\
\hline \multirow[t]{2}{*}{$\begin{array}{l}\text { Head (Below } \\
32 \mathrm{~cm} \text { ) }\end{array}$} & $32 \mathrm{~cm}$ to $36 \mathrm{~cm}$ & 0.422 & 0.119 & 0.002 & $\begin{array}{l}{[0.243 ;} \\
0.734]\end{array}$ & 0.422 & 0.115 & 0.002 & $\begin{array}{l}{[0.247 ;} \\
0.720]\end{array}$ & 0.422 & 0.118 & 0.002 & $\begin{array}{l}{[0.244 ;} \\
0.729]\end{array}$ \\
\hline & Above $36 \mathrm{~cm}$ & 0.246 & 0.187 & 0.065 & $\begin{array}{l}{[0.055 ;} \\
1.093]\end{array}$ & 0.246 & 3.784 & 0.927 & $\begin{array}{l}{[0.000 ;} \\
\left.3.030 \times 10^{12}\right]\end{array}$ & 0.246 & 0.251 & 0.169 & $\begin{array}{l}{[0.033 ;} \\
1.819]\end{array}$ \\
\hline \multirow[t]{2}{*}{$\begin{array}{l}\text { Height (Below } \\
36 \mathrm{~cm} \text { ) }\end{array}$} & $46 \mathrm{~cm}$ to $54 \mathrm{~cm}$ & 0.917 & 0.285 & 0.781 & $\begin{array}{l}{[0.499 ;} \\
1.687]\end{array}$ & 0.917 & 0.290 & 0.784 & $\begin{array}{l}{[0.494 ;} \\
1.704]\end{array}$ & 0.917 & 0.294 & 0.788 & $\begin{array}{l}{[0.489 ;} \\
1.721]\end{array}$ \\
\hline & Above $54 \mathrm{~cm}$ & 1.692 & 1.283 & 0.488 & $\begin{array}{l}{[0.383 ;} \\
7.476]\end{array}$ & 1.692 & 24.567 & 0.971 & $\begin{array}{l}{[0.000 ;} \\
\left.3.890 \times 10^{12}\right]\end{array}$ & 1.692 & 1.700 & 0.601 & $\begin{array}{l}{[0.236 ;} \\
12.140]\end{array}$ \\
\hline \multicolumn{6}{|c|}{ Adjusted MRSM } & \multicolumn{4}{|c|}{ Adjusted BMRSM } & \multicolumn{4}{|c|}{ Adjusted JMRSM } \\
\hline $\begin{array}{l}\text { Covariate } \\
\text { (reference) }\end{array}$ & Level & $H R$ & SE & $P>z$ & $95 \% \mathrm{Cl}$ & $H R$ & SE & $P>z$ & $95 \% \mathrm{Cl}$ & $H R$ & SE & $P>z$ & $95 \% \mathrm{Cl}$ \\
\hline \multirow[t]{2}{*}{$\begin{array}{l}\text { Age (Under } 20 \\
\text { years old) }\end{array}$} & $\begin{array}{l}20 \text { to } 34 \\
\text { years old }\end{array}$ & 0.262 & 0.092 & $p<0.001$ & $\begin{array}{l}{[0.132 ;} \\
0.522]\end{array}$ & 0.265 & 0.088 & $p<0.001$ & $\begin{array}{l}{[0.138 ;} \\
0.509]\end{array}$ & 0.265 & 0.088 & $p<0.001$ & $\begin{array}{l}{[0.138 ;} \\
0.508]\end{array}$ \\
\hline & $\begin{array}{l}35 \text { years old and } \\
\text { above }\end{array}$ & 0.407 & 0.155 & 0.018 & $\begin{array}{l}{[0.193 ;} \\
0.859]\end{array}$ & 0.421 & 0.151 & 0.016 & $\begin{array}{l}{[0.208 ;} \\
0.850]\end{array}$ & 0.421 & 0.146 & 0.013 & $\begin{array}{l}{[0.213 ;} \\
0.833]\end{array}$ \\
\hline \multirow[t]{2}{*}{$\begin{array}{l}\text { Abortion (Not } \\
\text { aborted) }\end{array}$} & Aborted once & 1.487 & 0.408 & 0.149 & $\begin{array}{l}{[0.868 ;} \\
2.546]\end{array}$ & - & - & - & - & - & - & - & - \\
\hline & $\begin{array}{l}\text { Aborted more } \\
\text { than once }\end{array}$ & 0.520 & 0.170 & 0.046 & $\begin{array}{l}{[0.274 ;} \\
0.987]\end{array}$ & -1.684 & -0.336 & -0.009 & $\begin{array}{c}-[1.138 ; \\
2.490]\end{array}$ & - & - & - & - \\
\hline Gender (Female) & Male & 1.764 & 0.297 & 0.001 & $\begin{array}{l}{[1.268 ;} \\
2.453]\end{array}$ & 0.322 & 0.097 & $p<0.001$ & $\begin{array}{l}{[0.178 ;} \\
0.583]\end{array}$ & 1.684 & 0.367 & 0.017 & $\begin{array}{l}{[1.098 ;} \\
2.582]\end{array}$ \\
\hline Number (Singleton) & Multiple & 0.308 & 0.101 & $p<0.001$ & $\begin{array}{l}{[0.162 ;} \\
0.586]\end{array}$ & 0.246 & 0.093 & $p<0.001$ & $\begin{array}{l}{[0.117 ;} \\
0.515]\end{array}$ & 0.322 & 0.101 & $p<0.001$ & $\begin{array}{l}{[0.175 ;} \\
0.594]\end{array}$ \\
\hline
\end{tabular}


Table 4 Efron estimation (Continued)

\begin{tabular}{|c|c|c|c|c|c|c|c|c|c|c|c|c|c|}
\hline \multirow{2}{*}{$\begin{array}{l}\text { MRSM } \\
\text { APGAR (Below } \\
4 / 10)\end{array}$} & \multirow[b]{2}{*}{$4 / 10$ to $6 / 10$} & \multirow[b]{2}{*}{0.249} & \multirow[b]{2}{*}{0.073} & \multirow[b]{2}{*}{$p<0.001$} & \multirow[b]{2}{*}{$\begin{array}{l}{[0.140 ;} \\
0.442]\end{array}$} & \multicolumn{2}{|c|}{ BMRSM } & \multirow[b]{2}{*}{$p<0.001$} & \multirow[b]{2}{*}{$\begin{array}{l}{[0.052 ;} \\
0.138]\end{array}$} & \multicolumn{3}{|c|}{ JMRSM } & \multirow[b]{2}{*}{$\begin{array}{l}{[0.110} \\
0.546]\end{array}$} \\
\hline & & & & & & 0.085 & 0.021 & & & 0.246 & 0.100 & 0.001 & \\
\hline & $7 / 10$ and above & 0.081 & 0.022 & $p<0.001$ & $\begin{array}{l}{[0.048 ;} \\
0.137]\end{array}$ & 0.225 & 0.057 & $p<0.001$ & $\begin{array}{l}{[0.137 ;} \\
0.369]\end{array}$ & 0.085 & 0.021 & $p<0.001$ & $\begin{array}{l}{[0.052 ;} \\
0.138]\end{array}$ \\
\hline \multirow[t]{2}{*}{$\begin{array}{l}\text { Weight (Under } \\
2500 \mathrm{~g} \text { ) }\end{array}$} & $\begin{array}{l}2500 \mathrm{~g} \text { to } \\
4500 \mathrm{~g}\end{array}$ & 0.222 & 0.057 & $p<0.001$ & $\begin{array}{l}{[0.135 ;} \\
0.366]\end{array}$ & 0.487 & 5.083 & 0.945 & $\begin{array}{l}{[0.000 ;} \\
\left.3.730 \times 10^{8}\right]\end{array}$ & 0.225 & 0.056 & $p<0.001$ & $\begin{array}{l}{[0.138,} \\
0.367]\end{array}$ \\
\hline & Above $4500 \mathrm{~g}$ & 0.430 & 0.276 & 0.189 & $\begin{array}{l}{[0.122 ;} \\
1.512]\end{array}$ & 0.403 & 0.105 & $p<0.001$ & $\begin{array}{l}{[0.242 ;} \\
0.671]\end{array}$ & 0.487 & 0.453 & 0.440 & $\begin{array}{l}{[0.078} \\
3.023]\end{array}$ \\
\hline \multirow[t]{3}{*}{$\begin{array}{l}\text { Head (Below } \\
32 \mathrm{~cm} \text { ) }\end{array}$} & $32 \mathrm{~cm}$ to $36 \mathrm{~cm}$ & 0.388 & 0.093 & $p<0.001$ & $\begin{array}{l}{[0.243 ;} \\
0.622]\end{array}$ & 0.252 & 3.678 & 0.925 & $\begin{array}{l}{[0.000 ;} \\
\left.6.680 \times 10^{11}\right]\end{array}$ & 0.403 & 0.108 & 0.001 & $\begin{array}{l}{[0.238} \\
0.683]\end{array}$ \\
\hline & Above $36 \mathrm{~cm}$ & 0.235 & 0.175 & 0.052 & $\begin{array}{l}{[0.054 ;} \\
1.014]\end{array}$ & & & & & 0.252 & 0.259 & 0.180 & $\begin{array}{l}{[0.034} \\
1.889]\end{array}$ \\
\hline & & \multicolumn{4}{|c|}{$x^{2}=203.061, p<0.001$} & \multicolumn{4}{|c|}{$x^{2}=172.14, p<0.001$} & \multicolumn{4}{|c|}{$x^{2}=21.514, p<0.001$} \\
\hline
\end{tabular}

covariates such as the parent's education level, employment and income; behavioral covariates namely smoking habit, alcohol consumption and dietary and physiotherapeutic variables such as sports activity level. These variables are not recorded in registry at KUTH.

\section{Conclusion}

Marginal Risk Set Model (MRSM) and related resampling using Bootstrap (BMRSM) and Jackknife (JMRSM) are described and compared with a use of the dataset on infant mortality. The JMRSM and MRSM displayed relatively close results. The risk is higher for babies whose parents are under 20 years old parents as compared to older parents. Babies born with APGAR greater or equal to $7 / 10$ were found to have a better survival outcome than those born with APGAR less than $4 / 10$ and those whose APGAR range between $4 / 10$ and $6 / 10$. The risk is lower for underweight babies as compared to babies with normal weight and overweight. The survival outcomes for babies with normal circumference of head were found to be better than those with relatively small head. The study suggests that pregnancy of under 20 years old parents should be avoided, also appropriate clinical ways of keeping pregnancy against any cause of infant abnormality could help in lowering infant mortality.

\section{Appendix}

\section{Bootstrap and Jackknife re-sampling methods}

\section{Bootstrap}

Consider the $p$ fixed covariates $\mathbf{x}_{i}=\left(x_{i 1}, x_{i 2}, \ldots, x_{i n}\right)$ in Eq. (2) where $x_{i} j, i \in[1, p]$ are independent and identically distributed possibly with distribution $F_{\theta}$ where $\theta$ is the statistical parameter of interest. Consider the distribution function $F_{R n}$ of a random variable $R_{n}\left(\mathbf{x}, F_{\theta}\right)$. A bootstrap method as described in [9], consists of generating samples.

$$
\mathbf{x}_{\boldsymbol{i}}{ }^{*}=\mathbf{x}_{\boldsymbol{i}}{ }^{* 1}, \mathbf{x}_{\boldsymbol{i}}{ }^{* 2}, \ldots, \mathbf{x}_{\boldsymbol{i}}{ }^{* B},
$$

where $\mathbf{x}_{i}{ }^{* k}, \mathrm{k} \in[1, B]$ are random samples of size $n$ drawn with replacement from the sample $\mathbf{x}_{i}$.

The variables of $\mathbf{x}_{i}{ }^{* k}$ are independent and identically distributed with distribution $\hat{F}_{\theta, n}$, given $\mathbf{x} ; \hat{F}_{\theta, n}$ is an estimator of $F_{\theta}$ from $\mathbf{x}_{i}$; $B$ is a number of bootstrap samples also known as replications.

The estimated standard error of the bootstrap statistic of interest is given in Efron and Tibshirani [9] as

$$
\hat{s} e_{B}=\sqrt{\frac{1}{B-1} \sum_{b=1}^{B}\left[\hat{\theta}^{*}(b)-\frac{1}{B} \sum_{b=1}^{B} \hat{\theta}^{*}(b)\right]^{2}}
$$

where $\hat{\theta}^{*}(b)$ is an estimate of the statistic of interest from the $b^{\text {th }}$ bootstrap sample,

$$
b=1,2, \ldots . B
$$

\section{Jackknife}

Consider the $p$ fixed covariates $\mathbf{x}_{i}=\left(x_{i 1}, x_{i 2}, \ldots, x_{i n}\right)$ in Eq. (2).

Let $\theta$ be a statistic of interest. The jackknife samples consist of leaving out one observation at a time, that is $n$ samples.

$$
\mathbf{x}_{i}{ }^{*}=\left(x_{i 1}, x_{i 2}, \ldots, x_{i k-1}, x_{i k+1}, \ldots, x_{i n}\right) \forall k \in[1, n][9] .
$$

The jackknife standard error estimate as propose [9], is

$$
\hat{s} e_{j a c k}=\sqrt{\frac{n-1}{n} \sum_{i=1}^{n}\left[\hat{\theta}^{*}(i)-\frac{1}{n} \sum_{i=1}^{n} \hat{\theta}^{*}(i)\right]^{2}}
$$

where $\theta^{*}(i), i \in[1, n]$ is a statistic of interest for the $i^{t h}$ jackknife sample. 
Table 5 Cox estimation

\begin{tabular}{|c|c|c|c|c|c|c|c|c|c|c|c|c|c|}
\hline \multicolumn{6}{|l|}{ MRSM } & \multicolumn{4}{|l|}{ BMRSM } & \multicolumn{4}{|c|}{ JMRSM } \\
\hline $\begin{array}{l}\text { Covariate } \\
\text { (reference) }\end{array}$ & Level & $\mathrm{HR}$ & SE & $P>z$ & $\begin{array}{l}95 \% \\
\mathrm{Cl}\end{array}$ & $\mathrm{HR}$ & SE & $P>z$ & $95 \% \mathrm{Cl}$ & $\mathrm{HR}$ & SE & $P>z$ & $95 \% \mathrm{Cl}$ \\
\hline \multirow[t]{2}{*}{$\begin{array}{l}\text { Age (Under } 20 \\
\text { years old) }\end{array}$} & $\begin{array}{l}20 \text { to } 34 \text { years } \\
\text { old }\end{array}$ & 0.193 & 0.085 & $p<0.001$ & $\begin{array}{l}{[0.081 ;} \\
0.458]\end{array}$ & 0.193 & 0.094 & 0.001 & $\begin{array}{l}{[0.074} \\
0.502]\end{array}$ & 0.193 & 0.088 & $p<0.001$ & $\begin{array}{l}{[0.079 ;} \\
0.472]\end{array}$ \\
\hline & $\begin{array}{l}35 \text { years old } \\
\text { and above }\end{array}$ & 0.267 & $\begin{array}{l}0.128 \\
p<0.001\end{array}$ & 0.006 & $\begin{array}{l}{[0.104 ;} \\
0.682]\end{array}$ & 0.267 & 0.131 & 0.007 & $\begin{array}{l}{[0.102 ;} \\
0.697]\end{array}$ & 0.267 & 0.124 & 0.004 & $\begin{array}{l}{[0.107 ;} \\
0.662]\end{array}$ \\
\hline Residence (Rural) & Urban & 0.766 & 0.150 & 0.175 & $\begin{array}{l}{[0.521 ;} \\
1.126]\end{array}$ & 0.766 & 0.221 & 0.356 & $\begin{array}{l}{[0.435 ;} \\
1.349]\end{array}$ & 0.766 & 0.221 & 0.356 & $\begin{array}{l}{[0.435 ;} \\
1.350]\end{array}$ \\
\hline $\begin{array}{l}\text { Antecedents (Not } \\
1 \text { st newborn) }\end{array}$ & 1st newborn & 0.763 & 0.185 & 0.264 & $\begin{array}{l}{[0.475 ;} \\
1.226]\end{array}$ & 0.763 & 0.219 & 0.345 & $\begin{array}{l}{[0.435 ;} \\
1.338]\end{array}$ & 0.763 & 0.194 & 0.289 & $\begin{array}{l}{[0.463 ;} \\
1.258]\end{array}$ \\
\hline \multirow[t]{2}{*}{$\begin{array}{l}\text { Abortion (Not } \\
\text { aborted) }\end{array}$} & Aborted once & 1.404 & 0.453 & 0.293 & $\begin{array}{l}{[0.746 ;} \\
2.643]\end{array}$ & 1.404 & 0.627 & 0.448 & $\begin{array}{l}{[0.585 ;} \\
3.369]\end{array}$ & 1.404 & 0.593 & 0.422 & $\begin{array}{l}{[0.613 ;} \\
3.215]\end{array}$ \\
\hline & $\begin{array}{l}\text { Aborted more } \\
\text { than once }\end{array}$ & 0.378 & 0.152 & 0.015 & $\begin{array}{l}{[0.172 ;} \\
0.830]\end{array}$ & 0.378 & 0.336 & 0.274 & $\begin{array}{l}{[0.066 ;} \\
2.155]\end{array}$ & 0.378 & 0.446 & 0.409 & $\begin{array}{l}{[0.038 ;} \\
3.814]\end{array}$ \\
\hline \multirow[t]{2}{*}{$\begin{array}{l}\text { Childbirth } \\
\text { (Ventouse) }\end{array}$} & Natural & 0.732 & 0.481 & 0.635 & $\begin{array}{l}{[0.202 ;} \\
2.653]\end{array}$ & 0.732 & 0.369 & 0.537 & $\begin{array}{l}{[0.273 ;} \\
1.968]\end{array}$ & 0.732 & 0.365 & 0.532 & $\begin{array}{l}{[0.276 ;} \\
1.945]\end{array}$ \\
\hline & Surgery & 1.016 & 0.654 & 0.980 & $\begin{array}{l}{[0.288 ;} \\
3.590]\end{array}$ & 1.016 & 0.480 & 0.973 & $\begin{array}{l}{[0.403 ;} \\
2.565]\end{array}$ & 1.016 & 0.455 & 0.971 & $\begin{array}{l}{[0.423 ;} \\
2.443]\end{array}$ \\
\hline Gender (Female) & Male & 1.991 & 0.405 & 0.001 & $\begin{array}{l}{[1.336 ;} \\
2.966]\end{array}$ & 1.991 & 0.534 & 0.010 & $\begin{array}{l}{[1.177 ;} \\
3.368]\end{array}$ & 1.991 & 0.601 & 0.023 & $\begin{array}{l}{[1.101 ;} \\
3.599]\end{array}$ \\
\hline $\begin{array}{l}\text { Number } \\
\text { (Singleton) }\end{array}$ & Multiple & 0.218 & 0.111 & 0.003 & $\begin{array}{l}{[0.080 ;} \\
0.589]\end{array}$ & 0.218 & 0.155 & 0.033 & $\begin{array}{l}{[0.054 ;} \\
0.882]\end{array}$ & 0.218 & 0.131 & 0.011 & $\begin{array}{l}{[0.067 ;} \\
0.709]\end{array}$ \\
\hline \multirow[t]{2}{*}{$\begin{array}{l}\text { APGAR (Below } \\
4 / 10 \text { ) }\end{array}$} & $4 / 10$ to $6 / 10$ & 0.080 & 0.042 & $p<0.001$ & $\begin{array}{l}{[0.029 ;} \\
0.224]\end{array}$ & 0.080 & 0.056 & $p<0.001$ & $\begin{array}{l}{[0.020 ;} \\
0.319]\end{array}$ & 0.080 & 0.052 & $p<0.001$ & $\begin{array}{l}{[0.022 ;} \\
0.287]\end{array}$ \\
\hline & $\begin{array}{l}\text { 7/10 and } \\
\text { above }\end{array}$ & 0.021 & 0.011 & $p<0.001$ & $\begin{array}{l}{[0.008 ;} \\
0.056]\end{array}$ & 0.021 & 0.014 & $p<0.001$ & $\begin{array}{l}{[0.006 ;} \\
0.076]\end{array}$ & 0.021 & 0.011 & $p<0.001$ & $\begin{array}{l}{[0.008 ;} \\
0.061]\end{array}$ \\
\hline \multirow[t]{2}{*}{$\begin{array}{l}\text { Weight (Under } \\
2500 \mathrm{~g} \text { ) }\end{array}$} & $\begin{array}{l}2500 \mathrm{~g} \text { to } \\
4500 \mathrm{~g}\end{array}$ & 0.236 & 0.070 & $p<0.001$ & $\begin{array}{l}{[0.131 ;} \\
0.423]\end{array}$ & 0.236 & 0.077 & $p<0.001$ & $\begin{array}{l}{[0.124 ;} \\
0.448]\end{array}$ & 0.236 & 0.068 & $p<0.001$ & $\begin{array}{l}{[0.134 ;} \\
0.415]\end{array}$ \\
\hline & Above $4500 \mathrm{~g}$ & 0.378 & 0.257 & 0.153 & $\begin{array}{l}{[0.100 ;} \\
1.436]\end{array}$ & 0.378 & 4.696 & 0.938 & $\begin{array}{l}{[0.000 ;} \\
\left.1.410 \times 10^{10}\right]\end{array}$ & 0.378 & 0.473 & 0.437 & $\begin{array}{l}{[0.033 ;} \\
4.386]\end{array}$ \\
\hline \multirow[t]{2}{*}{$\begin{array}{l}\text { Head (Below } \\
32 \mathrm{~cm} \text { ) }\end{array}$} & $\begin{array}{l}32 \mathrm{~cm} \text { to } 36 \\
\mathrm{~cm}\end{array}$ & 0.391 & 0.119 & 0.002 & $\begin{array}{l}{[0.216 ;} \\
0.708]\end{array}$ & 0.391 & 0.101 & $p<0.001$ & $\begin{array}{l}{[0.236 ;} \\
0.649]\end{array}$ & 0.391 & 0.115 & 0.001 & $\begin{array}{l}{[0.219 ;} \\
0.698]\end{array}$ \\
\hline & Above $36 \mathrm{~cm}$ & 0.212 & 0.171 & 0.055 & $\begin{array}{l}{[0.043 ;} \\
1.033]\end{array}$ & 0.212 & 3.376 & 0.922 & $\begin{array}{l}{[0.000} \\
\left.7.780 \times 10^{12}\right]\end{array}$ & 0.212 & 0.238 & 0.167 & $\begin{array}{l}{[0.023 ;} \\
1.913]\end{array}$ \\
\hline \multirow[t]{2}{*}{$\begin{array}{l}\text { Height (Below } \\
36 \mathrm{~cm} \text { ) }\end{array}$} & $\begin{array}{l}46 \mathrm{~cm} \text { to } 54 \\
\mathrm{~cm}\end{array}$ & 0.828 & 0.283 & 0.582 & $\begin{array}{l}{[0.423 ;} \\
1.620]\end{array}$ & 0.828 & 0.254 & 0.539 & $\begin{array}{l}{[0.454 ;} \\
1.512]\end{array}$ & 0.828 & 0.284 & 0.582 & $\begin{array}{l}{[0.423 ;} \\
1.622]\end{array}$ \\
\hline & Above $54 \mathrm{~cm}$ & 1.706 & 1.351 & 0.500 & $\begin{array}{l}{[0.361 ;} \\
8.060]\end{array}$ & 1.706 & 28.569 & 0.975 & $\begin{array}{l}{[0.000} \\
\left.3.090 \times 10^{14}\right]\end{array}$ & 1.706 & 1.747 & 0.602 & $\begin{array}{l}{[0.229 ;} \\
12.707]\end{array}$ \\
\hline \multicolumn{6}{|c|}{ Adjusted MRSM } & \multicolumn{4}{|c|}{ Adjusted BMRSM } & \multicolumn{4}{|c|}{ Adjusted JMRSM } \\
\hline $\begin{array}{l}\text { Covariate } \\
\text { (reference) }\end{array}$ & Level & $\mathrm{HR}$ & SE & $P>z$ & $\begin{array}{l}95 \% \\
\mathrm{Cl}\end{array}$ & $\mathrm{HR}$ & SE & $P>z$ & $95 \% \mathrm{Cl}$ & $\mathrm{HR}$ & SE & $P>z$ & $95 \% \mathrm{Cl}$ \\
\hline \multirow[t]{2}{*}{$\begin{array}{l}\text { Age (Under } 20 \\
\text { years old) }\end{array}$} & $\begin{array}{l}20 \text { to } 34 \text { years } \\
\text { old }\end{array}$ & 0.218 & 0.094 & $p<0.001$ & $\begin{array}{l}{[0.094 ;} \\
0.509]\end{array}$ & 0.219 & 0.078 & $p<0.001$ & $\begin{array}{l}{[0.109 ;} \\
0.439]\end{array}$ & 0.219 & 0.087 & $p<0.001$ & $\begin{array}{l}{[0.101 ;} \\
0.476]\end{array}$ \\
\hline & $\begin{array}{l}35 \text { years old } \\
\text { and above }\end{array}$ & 0.341 & 0.157 & 0.019 & $\begin{array}{l}{[0.138 ;} \\
0.841]\end{array}$ & 0.352 & 0.133 & 0.006 & $\begin{array}{l}{[0.167} \\
0.738]\end{array}$ & 0.352 & 0.141 & 0.009 & $\begin{array}{l}{[0.160 ;} \\
0.771]\end{array}$ \\
\hline \multirow[t]{2}{*}{$\begin{array}{l}\text { Abortion (Not } \\
\text { aborted) }\end{array}$} & Aborted once & 1.479 & 0.459 & 0.208 & $\begin{array}{l}{[0.804 ;} \\
2.719]\end{array}$ & - & - & - & - & - & - & - & - \\
\hline & $\begin{array}{l}\text { Aborted more } \\
\text { than once }\end{array}$ & 0.424 & 0.161 & 0.024 & $\begin{array}{l}{[0.201 ;} \\
0.892]\end{array}$ & -1.833 & - & -0.041 & $\begin{array}{r}-[1.025 ; \\
3.278]\end{array}$ & - & - & - & - \\
\hline Gender (Female) & Male & 1.886 & 0.374 & 0.001 & $\begin{array}{l}{[1.278 ;} \\
2.783]\end{array}$ & 0.227 & 0.136 & 0.013 & $\begin{array}{l}{[0.070 ;} \\
0.732]\end{array}$ & 1.833 & 0.528 & 0.036 & $\begin{array}{l}{[1.042 ;} \\
3.225]\end{array}$ \\
\hline $\begin{array}{l}\text { Number } \\
\text { (Singleton) }\end{array}$ & Multiple & 0.214 & 0.108 & 0.002 & $\begin{array}{l}{[0.079 ;} \\
0.576]\end{array}$ & 0.091 & 0.053 & $p<0.001$ & $\begin{array}{l}{[0.029 ;} \\
0.286]\end{array}$ & 0.227 & 0.135 & 0.013 & $\begin{array}{l}{[0.070 ;} \\
0.730]\end{array}$ \\
\hline
\end{tabular}


Table 5 Cox estimation (Continued)

\begin{tabular}{|c|c|c|c|c|c|c|c|c|c|c|c|c|c|}
\hline \multirow{2}{*}{$\begin{array}{l}\text { MRSM } \\
\text { APGAR (Below } \\
4 / 10 \text { ) }\end{array}$} & \multirow[b]{2}{*}{$4 / 10$ to $6 / 10$} & \multirow[b]{2}{*}{0.098} & \multirow[b]{2}{*}{0.050} & \multirow[b]{2}{*}{$p<0.001$} & \multirow[b]{2}{*}{$\begin{array}{l}{[0.036 ;} \\
0.267]\end{array}$} & \multicolumn{2}{|c|}{ BMRSM } & \multirow[b]{2}{*}{$p<0.001$} & \multirow[b]{2}{*}{$\begin{array}{l}{[0.010 ;} \\
0.067]\end{array}$} & \multicolumn{2}{|c|}{ JMRSM } & \multirow[b]{2}{*}{$p<0.001$} & \multirow[b]{2}{*}{$\begin{array}{l}{[0.024} \\
0.345]\end{array}$} \\
\hline & & & & & & 0.026 & 0.013 & & & 0.091 & 0.062 & & \\
\hline & $\begin{array}{l}\text { 7/10 and } \\
\text { above }\end{array}$ & 0.026 & 0.012 & $p<0.001$ & $\begin{array}{l}{[0.010 ;} \\
0.066]\end{array}$ & 0.215 & 0.060 & $p<0.001$ & $\begin{array}{l}{[0.125 ;} \\
0.371]\end{array}$ & 0.026 & 0.013 & $p<0.001$ & $\begin{array}{l}{[0.010 ;} \\
0.069]\end{array}$ \\
\hline \multirow[t]{2}{*}{$\begin{array}{l}\text { Weight (Under } \\
2500 \mathrm{~g} \text { ) }\end{array}$} & $\begin{array}{l}2500 \mathrm{~g} \text { to } \\
4500 \mathrm{~g}\end{array}$ & 0.213 & 0.057 & $p<0.001$ & $\begin{array}{l}{[0.125 ;} \\
0.361]\end{array}$ & 0.398 & 4.183 & 0.930 & $\begin{array}{l}{[0.000 ;} \\
\left.3.590 \times 10^{8}\right]\end{array}$ & 0.215 & 0.057 & $p<0.001$ & $\begin{array}{l}{[0.128 ;} \\
0.362]\end{array}$ \\
\hline & $\begin{array}{l}\text { Above } \\
4500 \mathrm{~g}\end{array}$ & 0.364 & 0.245 & 0.134 & $\begin{array}{l}{[0.097 ;} \\
1.364]\end{array}$ & 0.374 & 0.102 & $p<0.001$ & $\begin{array}{l}{[0.219 ;} \\
0.640]\end{array}$ & 0.398 & 0.385 & 0.340 & $\begin{array}{l}{[0.060 ;} \\
2.650]\end{array}$ \\
\hline \multirow[t]{3}{*}{$\begin{array}{l}\text { Head (Below } \\
32 \mathrm{~cm} \text { ) }\end{array}$} & $\begin{array}{l}32 \mathrm{~cm} \text { to } 36 \\
\mathrm{~cm}\end{array}$ & 0.349 & 0.090 & $p<0.001$ & $\begin{array}{l}{[0.211 ;} \\
0.579]\end{array}$ & 0.222 & 3.684 & 0.928 & $\begin{array}{l}{[0.000 ;} \\
\left.7.970 \times 10^{13}\right]\end{array}$ & 0.374 & 0.105 & $p<0.001$ & $\begin{array}{l}{[0.216 ;} \\
0.648]\end{array}$ \\
\hline & Above $36 \mathrm{~cm}$ & 0.199 & 0.160 & 0.044 & $\begin{array}{l}{[0.042 ;} \\
0.957]\end{array}$ & & & & & 0.222 & 0.253 & 0.186 & $\begin{array}{l}{[0.024 ;} \\
2.067]\end{array}$ \\
\hline & & \multicolumn{3}{|c|}{$x^{2}=200.400, p<0.001$} & & \multicolumn{4}{|c|}{$x^{2}=190.114, p<0.001$} & \multicolumn{4}{|c|}{$x^{2}=23.710, p<0.001$} \\
\hline
\end{tabular}

\section{Abbreviations}

AGM: Andersen-Gill Model; APGAR: Appearance, Pulse, Grimace, Activity and Respiration; BCPHM: Bootstrap Cox Proportional Hazards Model; BMRSM: Bootstrap Marginal Risk Set Model; CPHM: Cox Proportional Hazards Model; CPHM: Jackknife Cox Proportional Hazards Model; IMR: Infant Mortality Rate; JMRSM: Jackknife Marginal Risk Set Model; KUTH: Kigali University Teaching Hospital; MRSM: Marginal Risk Set Model; PWPM: Prentice, Williams and Peterson Model; SSA: Sub-Saharan Africa; WLWM: Wei, Lin and Weissfeld Model

\section{Acknowledgements}

We thank DELTAS Africa Initiative and the University of KwaZulu-Natal for jointly supporting this work.

\section{Authors' contributions}

SF-M participated in the study design and data acquisition and reviewed the study, S-R participated in the study conceptualization and reviewed the study, P-G collected the dataset, analysed the dataset and reported the text. All authors reviewed and approved the final manuscript.

\section{Funding}

Writing the manuscript is founded by the University of KwaZulu-Natal. The study design, data collection, data analysis and interpretation are funded by the Wellcome Trust, grant 107754/Z/15/Z-DELTAS Africa via Sub-Saharan Africa Consortium for Advanced Biostatistics (SSACAB).

\section{Availability of data and materials}

The dataset used is confidential. Some information on it is available from authors on reasonable request.

\section{Ethics approval and consent to participate}

The present study was approved by the Kigali University Teaching Hospital where dataset was taken from the hospital database, with consent that the names of both parents and children cannot be published.

\section{Consent for publication}

Not applicable.

\section{Competing interests}

The authors declare that they have no competing interests.

Received: 7 January 2019 Accepted: 24 January 2020

Published online: 10 February 2020

\section{References}

1. Ester PV, Torres A, Freire JM, Hernández V, Gil A. Factors associated to infant mortality in Sub-Saharan Africa. J Public Health Afr. 2011;2(e27):112-6.

2. Adetunji J, Bos ER. Disease and Mortality in Sub-Saharan Africa. The International Bank for Reconstruction and Development/ the World Bank. 2nd ed; 2006. URL http://www.ncbi.n/m.nih.gov/books/NBK2292/
3. Gatabazi P, Melesse SF, Ramroop S. Infant mortality at the Kigali University Teaching Hospital: Application of Aalen additive hazards model and comparison with other classical survival models. Afr Popul Stud. 2019;33(2): 4834-51.

4. Gatabazi P, Melesse SF, Ramroop S. Multiple events model for the infant at Kigali University teaching hospital. Open Public Health J. 2018:11:464-73.

5. Hamada C. Bootstrap Cox regression using SAS procedures. SAS Institute Japan Ltd. 1995:211:1258-62.

6. Utzet F, Sánchez A. Some applications of the bootstrap to survival analysis. Anuario de Psicologia. 1992;55:155-67.

7. Cook RJ, Lawless JF. The statistical analysis of recurrent events. New York: Springer; 2007.

8. Wei LJ, Glidden DV. An overview of statistical methods for multiple failure time data in clinical trials. Stat Med. 1997;16:833-9.

9. B Efron and R J Tibshirani. An introduction to the bootstrap. Chapman \& Hall/CRC, 1994.

10. Bělašková SB, Fišerová E, Krupičková S. Study of bootstrap estimates in Cox regression model with delayed entry. Mathematica. 2013;52(2):21-30.

11. Xu G, Sen B, Ying Z. Bootstrapping a change-point Cox model for survival data. Electron J Stat. 2014;8(1):1345-79.

12. Xiao L, Yao-Hua W, Dong-Sheng T. Jackknifed random weighting for Cox proportional hazards model. Sci China Math. 2012;55(4):775-86.

13. Gatabazi P, Melesse SF, Ramroop S. Re-sampled Cox proportional hazard model for the infant mortality at the Kigali University teaching hospital teaching hospital. Open Public Health J. 2019;12:136-44.

14. Wang Z, Wu Y, Zhao LC. Approximation by randomly weighting method in censored regression model. Sci China Ser A. 2009;52:567-76.

15. Zheng ZG. Random weighting method. Acta Math Appl Sin. 1987;10: 247-53.

16. Zheng ZG, Tu D. Random weighting method in regression models. Sci Sinica Series A. 1988;31:1442-59.

17. James LF. A study of a class of weighted bootstraps for censored data. Ann Stat. 1997;25:1595-621.

18. Quan H, Tsai WY. Jackknife for the proportional hazards model. J Stat Comput Simul. 1992;43:163-76.

19. Sauerbrei W, Schumacher M. A bootstrap resampling procedure for model building: application to the Cox regression model. Stat Med. 1992;11:2093-109.

20. Efron B. Censored data and the bootstrap. J Am Stat Assoc. 1981;76:312-9.

21. Hjort N. Bootstrapping Cox's regression model. Technical Report, Stanford University, Dept. of Statistics, vol. 241; 1985.

22. Kim J. Conditional bootstrap methods for censored data. PhD dissertation, Florida State University, Dept, of Statistics; 1990

23. Collet D. Modeling survival data in medical research. 2nd ed. London: Chapman \& Hall; 2003.

24. Wei $L J$, Lin DY, Weissfeld $L$. Regression analysis of multivariate incomplete failure time data by modelling marginal distributions. J Am Stat Assoc. 1989; 84(408):1065-73.

25. Gatabazi P, Kabera G. Survival analysis and its stochastic process approach with application to diabetes data; 2015. 
26. Sankaran $P G$, Anisha P. Shared frailty model for recurrent event data with multiple causes. J Appl Stat. 2011;38(12):2859-68.

27. Breslow NE. Covariance analysis of censored survival data. Biometrics. 1974; 30:89-99.

28. Efron B. The efficiency of Cox's likelihood function for censored data. J Am Stat Assoc. 1977;72:557-65.

29. Cox DR. Regression models and life-tables (with discussion). J R Stat Soc. 1972;34(2):187-220 Series B.

30. Parzen M, Lipsitz SR. A global goodness-of-fit statistic for Cox regression models. Biometrics. 1999;55:580-4.

31. Basinga P, Moore AM, Singh S, Remez L, Birungi F, Nyirazinyoye L. Unintended pregnancy and induced abortion in Rwanda: causes and consequences. New York: Guttmacher Institute; 2012

32. Olausson PO, Cnattinius S, Haglund B. Teenage pregnancies and risk of late fetal death and infant mortality. Br J Obstet Gynaecol. 1999;106:116-21.

33. Lampinen R, Julkunen KV, Kankkunen P. A review of pregnancy in women over 35 years of age. Open Nurs J. 2009;3:33-8.

34. Pongou R. Why is infant mortality higher in boys than in girls? A new hypothesis based on preconception environment and evidence from a large sample of twins. Demography. 2013;50:421-44.

35. Zarulli V, Jones JAB, Oksuzyanc A, Lindahl-Jacobsen R, Christensen K, Vaupel JW. Women live longer than men even during severe famines and epidemics. Proc Natl Acad Sci U S A. 2018;115(4):E832-40.

36. Monden CWS, Smits J. Mortality among twins and singletons in SubSaharan Africa between 1995 and 2014: a pooled analysis of data from 90 demographic and health surveys in 30 countries. Proc Natl Acad Sci U S A. 2017;5(7):E673-9

37. Pongou R, Shapiro D, Tenikue M. Mortality convergence of twins and singletons in sub-Saharan Africa. Demogr Res. 2019;41(36):1047-58.

38. Janssen PA, Thiessen P, Klein MC, Whitfield MF, Ying C, Cullis-Kuhl SC. Standards for the measurement of birth weight, length and head circumference at term in neonates of European, Chinese and South Asian ancestry. Open Med. 2007;1(2):74-88.

\section{Publisher's Note}

Springer Nature remains neutral with regard to jurisdictional claims in published maps and institutional affiliations.

Ready to submit your research? Choose BMC and benefit from:

- fast, convenient online submission

- thorough peer review by experienced researchers in your field

- rapid publication on acceptance

- support for research data, including large and complex data types

- gold Open Access which fosters wider collaboration and increased citations

- maximum visibility for your research: over $100 \mathrm{M}$ website views per year

At $\mathrm{BMC}$, research is always in progress.

Learn more biomedcentral.com/submissions 